Infolgedessen sind Individuen im öffentlichen wie auch im privaten Leben als Repräsentant_innen objektiver Positionen im sozialen Raum zu sehen (Blatter, Janning \& Wagemann, 2007). Für die Netzwerkforschung ist vor allem Bourdieus Betrachtung sowohl von Mikro- (Habitus) als auch Makrostrukturen (Feld) relevant. Der Strukturalismus der Netzwerkforschung wird somit durch die Perspektive sozialer Praktiken von Akteur_innen ergänzt. Die Struktur, in Form von unterschiedlichen Kapitalverteilungen, bestimmt massgeblich mit, wie sich subjektive Beziehungen konstruieren. Wesentlich für die Strukturierung des Feldes sind zudem Machtbeziehungen, die sich aus den unterschiedlichen Kapitalsorten ergeben und Gruppenprozesse auslösen können, im Sinne von Sieger_innen und Verlierer_innen innerhalb eines Feldes (Hennig \& Kohl, 2012, S. 21). Für die Netzwerkforschung ist weiter die Frage wesentlich, ob inkorporierte Denk- und Handlungsmuster (der Habitus) dazu führen, dass Akteur_innen vorwiegend innerhalb ihrer Klasse respektive mit ähnlichen sozialen Positionen interagieren. Der Habitus würde dann als Bindeglied innerhalb von Gruppen fungieren (Hennig \& Kohl, 2012).

\title{
IV.2 Relationen auf digitalen Plattformen
}

Wie im letzten Kapitel beschrieben, können klassisch soziologische Beiträge herangezogen werden, um Netzwerkdynamiken zu beschreiben. Dies ist für die vorliegende Arbeit von hoher Relevanz, zumal die digitale Öffentlichkeit Untersuchungsgegenstand ist, um das Phänomen von Gegenöffentlichkeiten zu analysieren. Zugrundeliegend ist die Annahme, dass digitale Öffentlichkeiten durch einen Netzwerkcharakter geprägt sind. Dies ist insbesondere im Zusammenhang mit der Untersuchung von Digitalplattformen, die einen wesentlichen Stellenwert in der Transformation der Öffentlichkeit einnehmen, relevant. Die folgenden Kapitel sollen aufzeigen, inwiefern digitale Öffentlichkeiten aktuell erforscht werden und wie diese Ansätze kritisch zu reflektieren sind (Kapitel IV.2.1). Abschliessend erfolgt eine Einordnung relational soziologischer Ansätze für die Erforschung von Digitalplattformen (Kapitel IV.2.2).

\section{IV.2.1 Aktuelle Ansätze zur Untersuchung digitaler Netzwerke ${ }^{5}$}

Obwohl die Erforschung von Netzwerken auf eine lange Tradition zurückblickt, hat sie in der empirischen Sozialforschung durch die Etablierung von Social-Media-Plattformen eine neue Rahmung erfahren. Populär wurde in diesem Zusammenhang vor allem die Analyse der Digitalplattform Twitter, die eine Datenerhe-

5 Dieses Kapitel bezieht sich vorwiegend auf Schwaiger (2021 [im Erscheinen]). 
bung mittels API-Schnittstellen ermöglicht (Bürger \& Dang-Anh, 2014). Twitter weist im Vergleich $\mathrm{zu}$ beispielsweise Facebook zwar geringere Nutzer_innenzahlen im deutschsprachigen Raum auf (Schwaiger, 2019), dennoch wird die Plattform zunehmend von Politiker_innen und politischen Journalist_innen/ Online-Nachrichten genutzt. Twitter wird daher auch als Elitennetzwerk bezeichnet (Ausserhofer \& Maireder, 2013; Rauchfleisch \& Metag, 2016). Eine Analyse der Twittersphäre mit Bezug auf Relationen von Medien und Politik (im Sinne der «öffentlichen Meinung») ist demzufolge naheliegend. Netzwerkanalytisch fokussiert werden am Untersuchungsgegenstand Twitter vor allem sogenannte «Follower-Netzwerke», also Nutzer_innengruppen, die gleichzeitig gemeinsame Interessenskonstellationen widerspiegeln, indem sie ähnliche Accounts auf der Plattform abonniert haben, oder auch «Retweet-Netzwerke», die Beziehungsrelationen darstellen, basierend auf geteilten Beiträgen unter den Akteur_innen (Schlögl \& Maireder, 2015). Verdichtungen (oder Communities) innerhalb eines Netzwerks können unter den Begriff der «Homophilie» gefasst werden, wonach Nutzer_innen einer Community über ähnliche Strukturen verfügen und im Vergleich zu anderen Communities z. B. ähnlichen Accounts folgen. Diese können wiederum als (Teil-)Öffentlichkeiten verstanden werden, «da sie sich aus der Artikulation von Interessen über die Auswahl von Followees ergeben, und da sie die Beobachtungs- und Handlungspotenziale für die Bezugnahmen der sozialen Akteure langfristig strukturieren» (Schlögl \& Maireder, 2015, S. 19). Entsprechend kann auch jede_r Nutzer_in Teil verschiedener Öffentlichkeiten sein, wobei sich bestimmte themenzentrierte Öffentlichkeiten beispielsweise über geteilte Hashtags identifizieren lassen.

Schlögl und Maireder (2015) analysieren in ihrer Studie beispielsweise die österreichische Twitterlandschaft und kommen zu dem Ergebnis, dass sich neben der Öffentlichkeit bzw. dem Cluster «Medien», in dem sich vor allem Journalist_ innen befinden, über die jeweiligen Follower_innen auch die politischen Parteien (SPÖ, ÖVP, FPÖ) recht trennscharf als Communities abbilden lassen. Bezugnehmend auf die Vernetzung zwischen Politik und Medien fanden Rauchfleisch und Metag (2016) heraus, dass in der Schweizer Twitterlandschaft besonders aktive Politiker_innen eine höhere Anzahl an Journalist_innen, die ihnen folgen, verzeichnen können. Dies ist auch deshalb interessant, da dies als ein Indiz für einen potentiellen Agenda-Building-Effekt ausgehend von Twitter gedeutet werden kann (Metag \& Rauchfleisch, 2016, 2017; Rauchfleisch \& Metag, 2016). Eine weitere beispielhafte Studie aus Deutschland zeigt - ähnlich dem Ergebnis aus Österreich -, dass auf Twitter eine Art Medien-Bubble existiert, in der sich Journalist_innen miteinander vernetzen und in ihren Inhalten stark an journalistischen Normen orientieren, aber auch eine Vernetzung mit politischen Akteur_innen vorhanden ist (wenn auch zu einem geringeren Teil) (Nuernbergk, 2016). Der Autor wertet dies als Indikator dafür, dass Twitter die Dynamiken zwischen Poli- 
tiker_innen und politischen Journalist_innen beeinflusst. Eine Twitter-Studie aus Dänemark, die sich mit ebendiesen Relationen zwischen Politik und Medien befasst, erhob den Befund, dass Communities von Journalist_innen und Politiker_innen nicht getrennt voneinander bestehen und entsprechend Informationen kontrollieren, sondern die jeweiligen Akteur_innen gegenseitig voneinander abhängig sind (Verweij, 2012). Eine weitere aktuelle Studie, die sich mit der Vernetzung von Nachrichtenmedien auf Twitter auseinandersetzt, zeigt am Beispiel der Schweizer Twittersphäre zudem, dass einzelne Communities (oder «Newsrepertoires«; vgl. Kapitel II.3.3) auch länderübergreifende Bezüge verzeichnen (Rauchfleisch, Vogler \& Eisenegger, 2020). Die Autoren belegen somit auf empirischer Basis, dass digitale Öffentlichkeiten von Globalisierungstendenzen geprägt sind (vgl. hierzu auch Castells, 2010 [1996]). Es handelt sich bei den genannten Studien ausschliesslich um beispielhafte Studien, die sich mit Nachrichtenmedien in der Twittersphäre netzwerkanalytisch auseinandersetzen. Gemein haben die Studien zudem (wie viele weitere Studien, die Digitalplattformen netzwerkanalytisch untersuchen) eine vorwiegend quantitative, computergestützte Herangehensweise an die Thematik. Interpretationen werden vor allem basierend auf statistischen Kennwerten vorgenommen, die die Netzwerkstrukturen näher beschreiben. In Anlehnung an relational soziologische Theorien könnten die Interpretationen der Strukturen weiter vertieft werden, was indes den Einsatz qualitativer Methoden implizieren würden.

Aus methodischer Sicht naheliegend, veränderte der digitale Wandel nicht nur die öffentliche Kommunikation, sondern auch die Möglichkeiten für Wissenschaftler_innen, die soziale Realität zu erforschen. So entwickelten sich nicht nur neue Forschungsfragen, sondern auch methodische Zugänge. Vor allem die Erforschung von digitalen Plattformen erwies sich dabei - nicht zuletzt aufgrund der dort auffindbaren Datenvielfalt und -menge - als ein beliebter Zugang für Sozialwissenschaftler_innen. Die Weiterentwicklung methodisch-analytischer Praktiken - passend auf die Untersuchungsfelder - führte in den vergangenen Jahren zu einem «Hype» so genannter «Computational Methods» oder «Big Data Analyses». In diesem Kontext veröffentlichte beispielsweise Anderson (2008) im «Wired»-Magazin einen Artikel mit dem Titel «The End of Theory» - digitale Methoden würden diesem Narrativ zufolge theoretische Annahmen und hypothetische Modelle obsolet machen, da durch neue, computergestützte Verfahren auch ohne diese Vorannahmen Muster in Daten abgelesen werden könnten, basierend auf Vollerhebungen. Diese Aussage in der Scientific Community blieb indes nicht unkritisiert (z. B. Bowker, 2014); Parks (2014) verwies in diesem Zusammenhang in zynischer Weise auf ein sich etablierendes «Big Data Movement».

Besonders die computergestützte Erforschung sozialer Netzwerkstrukturen bietet sich jedoch in diesem Zusammenhang erstens aufgrund der strukturellen Gegebenheit digitaler Plattformen an, die schliesslich als Netzwerke oder Relatio- 
nen zwischen Akteur_innen aufgebaut sind. Zweitens erscheint es auf den ersten Blick mehr als naheliegend, quantitative Verfahren für deren Analyse anzuwenden - nicht nur deshalb, weil die klassische Netzwerkforschung stark im quantitativen Paradigma verankert ist, sondern vor allem aufgrund der Datenfülle auf Digitalplattformen. Die dahinter liegende «grosse Zahl» würde die methodische Vorgehensweise potentiell vorgeben. Im Zuge von grösseren Forschungsarbeiten ist es jedoch von besonderer Relevanz, methodische Zugänge und Methodologien genau zu überdenken.

Neue, digitale Möglichkeiten der Datenerhebung und -auswertung sind dabei grundsätzlich als äusserst fruchtbar zu werten und bieten einen wesentlichen Mehrwert für die Erforschung von Digitalplattformen. Vor allem für die Untersuchung von Netzwerkstrukturen erweisen sich jene Zugänge als ergebnisträchtig, da diese Strukturen insbesondere durch eine quantitative und dennoch explorative Herangehensweise erst sichtbar werden. Kritisch zu hinterfragen ist dennoch eine zu starke Fokussierung auf ebendiese Methoden. So impliziert die Erhebung von Social-Media-Daten auch Restriktionen, wonach Wissenschaftler_innen von den Möglichkeiten, die von den Plattformen selbst gegeben werden, abhängig sind und gleichzeitig darauf vertrauen müssen, dass die generierten Daten tatsächlich jene Stichprobe abbilden, die auch angestrebt wurde. Werden beispielsweise Daten aufgrund bestimmter Restriktionen oder Algorithmen der Plattformen ausgeschlossen? Sind die erhobenen Datensätze vertrauenswürdig? Und implizieren grosse Datensätze tatsächlich eine grössere Erkenntnis (Lohmeier, 2014; Schwaiger, 2021 [im Erscheinen]).

Gleichzeitig kann festgehalten werden, dass immer mehr sozialwissenschaftliche Forscher_innen ebendiese Problematiken, aber auch Vorteile, die sich aus der computergestützten Erhebung von Social-Media-Daten ergeben, reflektieren. So sprechen sich immer mehr Soziolog_innen und Kommunikationswissenschaftler_innen dafür aus, dass eine Kombination computergestützter und klassischer sozialwissenschaftlicher Methoden häufig am zielführendsten sei (Bauhaus, 2016; Klemm \& Michel, 2014; Mahrt, 2016; Smith, 2014; Strippel et al., 2018). Schliesslich schafft die Darstellung von Daten- bzw. Netzwerkstrukturen zwar einen wesentlichen Mehrwert für die Deskription von Relationen, zu hinterfragen bleibt allerdings, inwiefern Narrative und der «subjektive Sinn» zwischen Akteur_innen (im Sinne der relationalen Soziologie) in derartige Analysen miteinbezogen werden können. Obgleich es auch auf inhaltlicher Ebene quantitative Auswertungsverfahren gibt (beispielsweise die computergestützte Analyse von «Sentiments»), gehe ich davon aus, dass latente Sinnstrukturen einen wesentlichen Bestandteil zwischen Relationen von Akteur_innen darstellen, die idealerweise mit interpretativen, qualitativen Verfahren zu analysieren sind. Gerade in diesem Bereich besteht noch Forschungsbedarf. Dabei sollte erstens der Anspruch einer theoretischen Einordnung oder auch Theoriebildung wesentlich 
für dahingehende Untersuchungen sein. Die Annahme einer «sterbenden Theorie» (Anderson, 2008) - im Sinne einer fehlenden Relevanz theoretischer Arbeit - aufgrund der Auswertung grosser (vollumfänglicher) Datensätze würde dabei meines Erachtens gegen jeglichen wissenschaftlichen Auftrag verstossen. Vielmehr wurde in Kapitel IV.1 deutlich, dass gerade bei der Erforschung von Netzwerkstrukturen klassisch theoretische Schriften fruchtbar sind, um die Rolle von Beziehungsstrukturen in der Gesellschaft erst einordnen zu können. Theoriebildung ist weiter wesentlich für wissenschaftlichen Fortschritt. Ausserdem müssen Datenstrukturen erst mit «Sinn» gefüllt werden, um beurteilen zu können, was hinter manifesten Inhalten steht. Hierfür eignen sich vor allem qualitative Methoden (vgl. hierzu auch Mahrt, 2016).

Aus diesen Gründen wird in der vorliegenden Arbeit bewusst auf die Kombination computergestützter quantitativer Verfahren und qualitativer Auswertungstechnik für die Interpretation von Relationen zwischen Akteur_innen gesetzt. Unter Rückbezug auf die erörterten klassischen Schriften, die der relationalen Soziologie zugeordnet werden können (z. B. Simmel, Bourdieu, Elias), wird versucht, die Vorteile aktueller methodischer Zugänge mit traditionellen Verfahren $\mathrm{zu}$ verknüpfen. Als wesentlich erachtet wird eine genaue Untersuchung dessen, was hinter starren Strukturen (z. B. in Form von Netzwerkstrukturen) liegt, um den subjektiven Sinn von Akteur_innen in Erfahrung zu bringen. Dabei handelt es sich um eine Kombination der Beschreibung von Netzwerkdynamiken und dem Verstehen dieser (Diaz-Bone, 2008).

\section{IV.2.2 Relational soziologische Ansätze und ihre Anwendbarkeit auf die Untersuchung von digitalen Öffentlichkeiten}

Im folgenden Kapitel werden theoretische Annahmen hinsichtlich der Erforschung von Digitalplattformen reflektiert. Die drei beschriebenen Ansätze von Simmel (1968 [1908]), Elias (2006 [1970]) und Bourdieu (2013 [1979]) bieten dabei einen metatheoretischen Rahmen, um Netzwerkstrukturen interpretativ einordnen zu können. Bezogen auf das Untersuchungsfeld von Digitalplattformen können beispielsweise Simmels (1968 [1908]) soziale Kreise als Äquivalent für die Entstehung von Communities in sozialen Netzwerken betrachtet werden. Die individuellen Sets sozialer Kreise basieren vor allem auf den Wechselwirkungen zwischen Individuen, Beziehungen entstehen in erster Linie basierend auf ähnlichen Neigungen, Tätigkeiten, Interessen, aber auch auf Zweck und Rationalisierung, die eine Homophilie befördern. Das Konzept der Homophilie ist auch in der modernen Netzwerkforschung zentral. So werden Communities auf Digitalplattformen z. B. basierend auf ihren «Followerstrukturen» gebildet. Strukturell betrachtet wird ein ähnliches Verhalten von Social-Media-User_innen, z. B. durch das Abonnieren oder Teilen von Beiträgen ähnlicher Accounts, mit der Gegeben- 
heit ähnlicher Interessen gleichgesetzt. Ein sozialer Kreis entstünde demnach bereits durch das Vernetzen mit unterschiedlichen Akteur_innen, wobei die Individualität einzelner User_innen gleichbedeutend auf den individuellen Sets an Kontakten innerhalb der Digitalplattform beruhen würde. Je nach Community nähmen einzelne Akteur_innen zentralere oder peripherere Stellungen ein. Auch diese Zentralität könnte rein statistisch in der Netzwerkforschung analysiert werden. Wesentlich wird Simmels (1968 [1908]) Ansatz vor allem dann, wenn der Kern seiner Theorie genauer betrachtet wird. Relationen beziehen sich demzufolge vor allem auf wechselseitige Erwartungen zwischen Individuen, die einen subjektiven Sinn hinter Beziehungen implizieren. Diese würden, einem strukturalistischen Ansatz von Netzwerken folgend, nicht hinterfragt werden. Insofern würde durch das blosse Betrachten von quantitativen Gesichtspunkten, wie der Anzahl an Follower_innen oder Retweets, um starke Knoten innerhalb der Netzwerke zu identifizieren, nicht in Erfahrung bringen, warum sie einen zentralen Stellenwert im Netzwerk einnehmen. Zudem kann nur durch eine inhaltliche, interpretative Analyse der Äusserungen von Individuen (z. B. in Form von Beiträgen, die auf Digitalplattformen veröffentlicht werden) untersucht werden, worauf sich Relationen zwischen Akteur_innen begründen, beispielsweise basierend auf Rationalität, gemeinsamen Interessen, oder eben ganz im Gegenteil: basierend auf Gegenmeinungen, um die Erwartungen innerhalb der eigenen Community (dem eigenen sozialen Kreis) zu erfüllen.

Als zentral betrachtet auch Elias (2006 [1970]) die wechselseitigen Bezugnahmen von Individuen - im Sinne von Figurationen - für die Gemeinschaftsbildung und nimmt damit Abstand von einem ego-zentrierten Fokus auf Individuen. Da diese situationsbedingt wechseln können, sind auch Netzwerkstrukturen keine starren Gebilde. Infolgedessen wäre es denkbar, dass öffentlich kommunizierte Themen Transformationen im Netzwerk bedingen. Gegenöffentlichkeiten könnten in diesem Kontext neue Themen setzen und zunehmende Bedeutung erlangen respektive sich zu grösseren Teilöffentlichkeiten (im Sinne von Communities) transformieren. Vor allem Machtbalancen, die auf den jeweiligen Interdependenzen von Individuen beruhen und nicht per se für bestimmte Akteur_innen zutreffen, nehmen in Elias' (2006 [1970]) Figurationsansatz eine wesentliche Rolle ein. Wird dieses Bild auf die Netzwerke von Digitalplattformen adaptiert, könnte davon ausgegangen werden, dass zentrale Knoten innerhalb von Netzwerken über besondere Deutungsmacht verfügen. Dies basiert aber weniger auf gegebenen Ressourcen, die Macht verleihen, sondern vielmehr auf den Beziehungen, die innerhalb des Netzwerks aufgebaut werden und bestimmten Akteur_innen eine höhere Macht zuschreiben (z. B. indem Beiträge bestimmter Akteur_innen durch andere User_innen weiter gestreut und deren Reichweiten erhöht werden, als Zeichen einer zustimmenden Haltung). Elias (2006 [1970]) spricht weiter von dynamischen Machtverhältnissen, wonach sich Macht und Gegenmacht gegen- 
überstünden. Konkret setzt sich der Autor mit dieser Thematik in seinen Ausführungen zu Etablierten und Aussenseiter_innen auseinander (Elias \& Scotson, 2002 [1965]). Diese Gegenmächte und Ingroup- respektive Outgroup-Dynamiken sind besonders relevant im Kontext von Öffentlichkeit und Gegenöffentlichkeit respektive öffentlichen und gegenöffentlichen Gruppen. Es ist zu erwarten, dass ebendiese Dynamiken Digitalplattformen mitprägen.

Abschliessend offenbart Bourdieus (2013 [1979]) Habitus- und Feldtheorie genau jene Herausforderung, die die Erforschung von Netzwerkstrukturen mit sich bringt. Bourdieu fokussiert einerseits die Makroperspektive, im Sinne von strukturellen Gegebenheiten, die sich im sozialen Raum ergeben. Auf der Mikroperspektive gibt der individuelle Habitus Aufschluss darüber, in welcher Form Beziehungen zu interpretieren sind. Zentral sind bei Bourdieu zudem die jeweiligen Kapitalverteilungen, die die Position im sozialen Feld definieren. In Abhängigkeit von ökonomischen, kulturellen und sozialen Ressourcen ergibt sich die jeweilige Position. So erscheint bei der Analyse von sozialen Netzwerken im Sinne von Digitalplattformen insbesondere das Sozialkapital als wesentlich. Die Bedeutung von Sozialkapital verdeutlicht sich mit Blick auf die Logiken der Plattformen, die eine Vernetzung zwischen Akteur_innen durch gegenseitiges Folgen anregen. Je höher das soziale Kapital (oder je grösser die Anzahl an Abonnent_innen), desto stärker die Position im digitalen Netzwerk. Gleichzeitig ergibt sich daraus das symbolische Kapital, indem Akteur_innen diese zentralen Knoten wahrnehmen und unterstützen (beispielsweise durch Retweets von deren Beiträgen). Wie Individuen auf den Plattformen agieren, welche Beiträge sie verfassen und teilen, gibt zudem Aufschluss über den individuellen Habitus und darüber, zu welcher Gruppe sie sich zugehörig fühlen. Der Habitus wirkt entsprechend gruppenbildend.

Dieses Kapitel hat mögliche Ansätze vorgestellt, wie Netzwerkrelationen interpretiert werden können. Dies erscheint vor allem für die Analyse von Digitalplattformen, die von Netzwerkstrukturen geprägt sind, ergebnisträchtig. Deutlich wurde zudem, dass ein Blick auf Sinnstrukturen, die hinter den Relationen liegen, wesentlich ist, um Dynamiken in öffentlichen und gegenöffentlichen Netzwerken interpretieren zu können. Für die vorliegende Arbeit wurde daher ein interpretativer Zugang gewählt, um die Relationen innerhalb von Digitalplattformen zu interpretieren. 
